ISSN 0103-9954

\title{
ANÁLISE DE SOBREVIVÊNCIA E CRESCIMENTO DE Cordia trichotoma, BORAGINACEAE, LAMIALES, NO SUL DE MATO GROSSO DO SUL - BRASIL
}

\author{
SURVIVAL ANALYSIS AND GROWTH OF Cordia trichotoma, BORAGINACEAE, LAMIALES, IN \\ MATO GROSSO DO SUL STATE, BRAZIL
}

\author{
Sergio Luiz Salvadori ${ }^{1}$ Claudia Universal Neves Batista Deinzer Duarte ${ }^{2}$ \\ Ana Francisca Gomes da Silva ${ }^{3}$ Wagner Lopes Klein ${ }^{4}$
}

\begin{abstract}
RESUMO
A avaliação da sobrevivência e do crescimento de um vegetal pode refletir sua habilidade competitiva na comunidade vegetal. Cordia trichotoma é uma árvore nativa comum no estado de Mato Grosso do Sul, e uma das mais promissoras para plantio. O presente trabalho monitorou o percentual de sobrevivência e crescimento de Cordia trichotoma em condições diferentes no quesito capina e quanto ao recebimento ou não de adubação. O trabalho teve início em setembro de 2008 e término em março de 2010 . A coleta e semeadura foram realizadas em área urbana do município de Mundo Novo e a área para plantio definitivo, para mensuração da sobrevivência e crescimento das mudas, no município de Japorã, fazenda Santa Clara. As mudas foram plantadas e divididas em duas categorias de acordo com o uso de adubo, podendo ter ou não coroamento resultando em quatro grupos distintos: bloco adubo terra nua (ATN), bloco sem adubo terra nua (BTN), bloco adubo e coroado (AC) e bloco sem adubo e coroado (BC). Os resultados indicaram alta sobrevivência de Cordia trichotoma no sistema de transplante de canteiro para sacos de mudas. O bloco BC apresentou maior percentual de sobrevivência, porém, menor incremento em altura. Já os blocos AC, ATN e BTN revelaram o mesmo padrão de sobrevivência, além de média de crescimento semelhante. Entretanto, podem existir diferenças nutricionais e químicas na composição do solo sugerindo análises setoriais em estudos futuros.
\end{abstract}

Palavras-chave: monitoramento de plantas; louro-pardo; Mundo Novo-MS.

\section{ABSTRACT}

The evaluation of a plant survival percentage and growth may reflect its competitive ability in plant community. Cordia trichotoma is a common native tree in Mato Grosso do Sul State and one of the most promising for planting. This study monitored the survival percentage and growth of Cordia trichotoma under different conditions such as weeding and receiving or not fertilization. The experiment started in September 2008 and it was concluded in March 2010. The seeds collection and sowing were held in urban area of Mundo Novo Municipality and the area for permanent planting to measure seedlings survival and growth was set at Japorã Municipality, Fazenda Santa Clara. Seedlings were planted in two categories: the use or not of fertilizer and crowing resulting in four distinct groups: block fertilizer bare earth (ATN), bare land block without fertilizer (BTN), fertilizer and crown block (AC) and without fertilizer and crowned block (BC). The results indicated high survival of Cordia trichotoma in the seedling transplant system from

1 Biólogo, Universidade Estadual de Mato Grosso do Sul, Unidade Universitária de Mundo Novo, Grupo de Estudos em Ciências Ambientais e Educação (GEAMBE), BR 163, Km 20,2, CEP 79980-000, Mundo Novo (MS), Brasil.

2 Bióloga, Msc., Professora da Universidade Estadual de Mato Grosso do Sul, Unidade Universitária de Mundo Novo, Grupo de Estudos em Ciências Ambientais e Educação (GEAMBE), BR 163, Km 20,2, CEP 79980-000, Mundo Novo (MS), Brasil. claudia.universal@gmail.com

3 Química, Dr ${ }^{\text {a }}$, Professora da Universidade Estadual de Mato Grosso do Sul, Unidade Universitária de Mundo Novo, Grupo de Estudos em Ciências Ambientais e Educação (GEAMBE), BR 163, Km 20,2, CEP 79980-000, Mundo Novo (MS), Brasil.

4 Matemático, Msc., Professor da Universidade Estadual de Mato Grosso do Sul, Unidade Universitária de Mundo Novo, Grupo de Estudos em Ciências Ambientais e Educação (GEAMBE), BR 163, Km 20,2, CEP 79980-000, Mundo Novo (MS), Brasil.

Recebido para publicação em 08/09/2011 e aceito em 13/08/2012 
bed to bags. The BC block showed the highest percentage of survival, but the smaller increments in height. The AC, ATN and BTN blocks presented the same survival pattern and similar average growth. However, there may be differences in nutritional and chemical composition of the soil suggesting sector analysis for future studies.

Keywords: monitoring plants; louro-pardo; Mundo Novo, Mato Grosso do Sul state.

\section{INTRODUÇÃO}

O desflorestamento traz como consequência a eliminação da vegetação natural e com ela a diminuição e até mesmo o desaparecimento da biota, além da modificação do microclima e, agora a nível mundial, do macroclima. Mas além dessas alterações no ecossistema, o desmatamento seguido pelo manejo inadequado do solo tem levado à destruição dos recursos naturais (WISH et al., 2008).

Barbosa (2000) afirma que os recursos renováveis, como: solo, água, fauna e vegetação, são fatores de desenvolvimento para o país quando manejados adequadamente. Para Crestana (2007), todo local em que a vegetação foi eliminada é possível reverter a situação por meio de diversos processos de recuperação de florestas. Segundo o mesmo autor, o período necessário para essa recomposição depende diretamente do nível de devastação que a vegetação original sofreu, ou seja, do estágio de perturbação no qual se encontra, e principalmente das condições de degradação do solo que as suportam.

Em processos de recomposição vegetal podem ser avaliados percentuais de sobrevivência e crescimento de mudas a serem implantadas, o que pode gerar maior produtividade e economia. As espécies florestais, de maneira geral, apresentam características distintas para o percentual de sobrevivência e para o crescimento, por isso a necessidade de se avaliar esses comportamentos (SOUZA et al., 2006).

Cordia trichotoma (Vell.) Arrabida ex Steudel, conhecida como Louro-Pardo, é uma planta arbórea de crescimento oportunista com dispersão natural por anemocoria e que pode alcançar até $35 \mathrm{~m}$ de altura, com tronco reto e cilíndrico, dando fustes de 10 a $20 \mathrm{~m}$ de altura (SCHEEREN et al., 2002; CARVALHO, 1988a). A área de dispersão estende-se da Floresta Tropical Pluvial Atlântica, do Sul da Bahia e norte do Espírito Santo, até a Floresta Subtropical Pluvial das bacias dos rios Paraná, Paraguai, Argentina e Uruguai (SCHEEREN et al., 2002; LORENZI, 2002).
Além de ser uma espécie nativa promissora para plantio, apresenta uma combinação de aspectos favoráveis, como rápido crescimento, frutificação abundante, regeneração natural vigorosa e facilidade de produção de mudas (CARVALHO, 1988a). A espécie revelou ainda ser eficiente no enriquecimento de bosques nativos, sendo cultivada em pequena escala por agricultores (CARVALHO, 1988b; EMBRAPA, 1986).

Inserido neste contexto, neste trabalho avaliou-se o crescimento e percentual de sobrevivência de mudas de Cordia trichotoma, apurando o desempenho desta espécie em diferentes situações de campo, através da realização do monitoramento do percentual de sobrevivência de mudas transplantadas de canteiros para saquinhos plásticos e das mudas para locais definitivos, bem como, a mensuração do incremento de altura em condições de plantios diferentes, quanto às condições de tratos culturais no quesito capina e ao recebimento ou não de adubação. Desta forma, este trabalho pode fornecer dados que venham contribuir no uso da Cordia trichotoma em reflorestamentos, como também o cultivo para uso comercial, além de subsidiar trabalhos futuros nesta área.

\section{MATERIAIS E MÉTODOS}

\section{Área de estudo}

O plantio das sementes ocorreu numa área de $150 \mathrm{~m}^{2}$ no município de Mundo Novo-MS, (S $23^{\circ} 56,009^{\prime}$; WO $54^{\circ} 17,155^{\prime}$ ), onde foi realizado o acompanhamento de sobrevivência de 600 mudas, o transplante e a seleção de 200 mudas para plantio definitivo.

A área a ser reflorestada com as 200 mudas selecionadas para plantio definitivo tem $1300 \mathrm{~m}^{2}$ e está localizada na Fazenda Santa Clara (S 2348,770'; WO 54²9,894'), no município de Japorã, MS. Nesse local foi feito o acompanhamento do percentual de sobrevivência e do crescimento das mudas, mediante diferentes condições de capinas e adubação. 
O município de Japorã-MS apresenta clima úmido com precipitação anual entre 1750 e 2000 mm; clima tropical de inverno seco (temperaturas inferiores a $18^{\circ} \mathrm{C}$ ) e verão quente (temperaturas superiores a $22^{\circ} \mathrm{C}$ ) (MATO GROSSO DO SUL, 2009). Apresenta solos predominantemente hidromórficos com texturas arenosa e argilosa. A vegetação primitiva desta área era basicamente de diversas formações de Floresta Estacional e Semidecidual (SEMAC/MS, 2010), onde hoje se encontra apenas fragmentos dessas formações.

\section{Coleta e semeadura das sementes}

A coleta das sementes ocorreu em julho de 2008, de Cordia trichotoma, com 15 metros de altura, existente no mesmo local onde foi preparado o canteiro (Mundo Novo-MS). Essa coleta foi realizada com o auxílio de um aspirador de pó.

As sementes foram plantadas em setembro de 2008, sem qualquer tratamento pré-germinativo. As plântulas ficaram em local com menor incidência de sol conforme descrito em Melo et al. (1998) e contra o frio (CARVALHO, 1988a).

\section{Formação das mudas}

Em janeiro de 2009, após 120 dias do plantio, foram selecionadas aleatoriamente 600 mudas e transplantadas para sacos plásticos próprios, com tamanho $13 \times 15 \mathrm{~cm}$, foram irrigadas e tiveram suas raízes pivotantes cortadas logo abaixo de um pequeno bulbo que se forma nesta raiz.

Após 120 dias do transplante das 600 mudas, foi realizada a contagem do percentual de sobrevivência (PS), das quais foram selecionadas para plantio definitivo 200 mudas com aproximadamente $13 \mathrm{~cm}$ de altura e para verificação do PS destas mudas, foram feitas contagens com 30, 180 e 300 dias após o plantio. Tais mudas não foram submetidas a processos de rustificação quanto à quantidade de regas, nem quanto à exposição à luminosidade.

\section{Preparação do terreno}

O plantio definitivo ocorreu em terreno previamente preparado mecanicamente, com auxílio de um trator e grade de arrasto, onde foi distribuído veneno granulado para formigas cortadeiras na área e nas suas proximidades. Com base nos resultados obtidos na análise química, o solo foi revolvido e não foi feita adubação e correção.

\section{Plantio em local definitivo}

Em maio de 2009, as 200 mudas foram plantadas aleatoriamente formando 4 grupos conforme as Figuras 1, 2, 3 e 4. O espaçamento foi de $1,8 \mathrm{~m}$ entre plantas na mesma linha e de $3 \mathrm{~m}$ entre as linhas.

\section{Divisões da área de plantio definitivo}

A área de plantio definitivo (parcela) foi dividida em 2 categorias, segundo a utilização ou não de adubo (Figura 1).

\begin{tabular}{|l|l|}
\hline BLOCO A & BLOCO B \\
\hline
\end{tabular}

FIGURA 1: Divisão da parcela quanto à adubação. Bloco A - Parcela com 100 mudas fertilizadas com adubo químico; Bloco B - Parcela com 100 mudas sem fertilizantes.

FIGURE 1: Parcel division regarding to fertilization. Treatment A - Parcel with 100 seedlings fertilized with chemical fertilizer; Treatment B - Parcel with 100 seedlings without chemical fertilizer.

\section{Divisões da parcela referente ao modo de capina}

O Bloco A foi subdividido em dois sub-blocos (Figura 2) sendo eles: Adubada e Terra Nua (Bloco ATN) - recebeu capina periódica na parcela toda mantendo o bloco livre de competição, ou seja, capinas constantes, inclusive nas entrelinhas; Adubada e Coroada (Bloco AC) - recebeu capina periódica de coroamento num raio de $30 \mathrm{~cm}$ em torno das mudas.

\begin{tabular}{|c|c|}
\hline $\begin{array}{c}\text { ADUBADO E TERRA } \\
\text { NUA (ATN) }\end{array}$ & ADUBADO E \\
COROADO (AC)
\end{tabular}

FIGURA 2: Divisão da Parcela, quanto ao método de capina em dois Blocos com adubação: ATN - 50 mudas e AC - 50 mudas coroadas.

FIGURE 2: Parcel Division, the method of weeding in two blocks with fertilization: ATN - 50 seedlings and AC - 50 crowned seedlings. 
O Bloco B também foi subdividido em dois sub-blocos conforme a Figura 3: Sem adubo e Terra Nua (Bloco BTN) - recebeu capina periódicas em toda parcela; Sem adubo e Coroado (Bloco BC) recebeu capina de coroamento num raio de $30 \mathrm{~cm}$ em torno das mudas.

\begin{tabular}{|c|c|}
\hline $\begin{array}{c}\text { SEM ADUBO E TERRA } \\
\text { NUA }(\mathrm{BTN})\end{array}$ & SEM ADUBO E \\
COROADO $(\mathrm{BC})$
\end{tabular}

FIGURA 3: Divisão da Parcela, quanto ao método de capina em dois Blocos sem adubação: BTN - 50 mudas e BC - 50 mudas coroadas.

FIGURE 3: Parcel Division, the method of weeding in two blocks without fertilization: BTN - 50 seedlings and BC - 50 crowned seedlings.

Desta forma, a área ficou representada conforme a Figura 4.

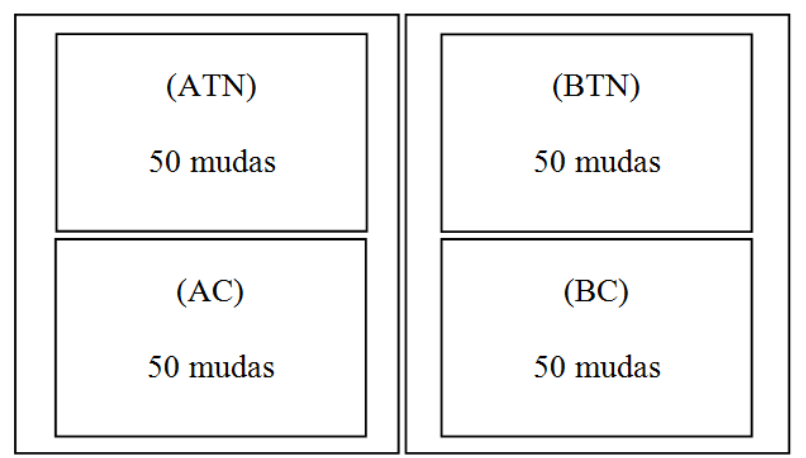

FIGURA 4: Divisões da área em parcelas.

FIGURE 4: Divisions of the area in installments.

\section{Adubação}

Nas mudas plantadas definitivamente, o adubo químico utilizado foi NPK 4-20-20, na proporção de $100 \mathrm{~g} /$ planta, aplicado uma única vez, 120 dias após o plantio no local definitivo.

\section{Avaliação do incremento}

O incremento da parte aérea do caule até a extremidade superior foi obtido 30 dias após o plantio no local definitivo, e as demais medidas foram realizadas trimestralmente utilizando uma trena metálica. Efetuaram-se quatro medições de altura das plantas na parcela 1 , sendo o primeiro incremento a diferença entre a segunda medida da altura e a primeira medida; o segundo incremento, a diferença entre a terceira e a segunda medida e o terceiro incremento a diferença entre a quarta e a terceira medida.

\section{Análise estatítica}

Para realização da análise dos dados utilizou-se a estatística descritiva, onde foram calculados: média, mediana, desvio padrão, coeficiente de variação, quartis, curtose e assimetria, visando identificar e avaliar se os dados possuíam homogeneidade e normalidade entre os tratamentos de Cordia trichotoma. O coeficiente de variação (CV) foi considerado baixo, quando $\mathrm{CV}<10 \%$ (homocedasticidade); médio, quando $10 \%<\mathrm{CV}<20 \%$; alto, quando $20 \%<\mathrm{CV}<30 \%$; e muito alto, quando $\mathrm{CV}$ $>30 \%$ (heterocedasticidade) (GOMES, 2002).

\section{RESULTADOS E DISCUSSÃO}

O percentual de sobrevivência das 600 mudas transplantadas para os saquinhos foi de $97 \%$ com poda da raiz pivotante, indicando tolerância ao transplante para mudas de Cordia trichotoma com até 240 dias, em condições de sombra e rega diária.

Para as mudas em locais definitivos, os resultados obtidos em relação ao percentual de sobrevivência em cada Bloco, com seus respectivos períodos, estão apresentados na Tabela 1 .

Todas as mudas dos diferentes blocos foram plantadas recebendo a mesma incidência de radiação solar e na ausência de competitividade. Após 30 dias de plantio já se encontravam em situações diferentes de competitividade por nutrientes e água, visto que $\mathrm{AC}$ e $\mathrm{BC}$ receberam capinas de coroamento. Espécies ou população de uma espécie que crescem próximas apresentam interações positivas e negativas. A competição é uma interação negativa, na qual os organismos envolvidos utilizam um recurso em suprimento escasso, resultando em prejuízo mútuo ao crescimento (RADOSEVICH et al., 1997).

Quando a quantidade de radiação solar não é limitante, como acontece nos estágios iniciais de desenvolvimento, podem ocorrer alterações nas plantas devido à competição (CARVALHO, 1988a; BALLARÉ e CASAL, 2000; ALMEIDA e MUNDSTOCK, 2001). 
TABELA 1: Percentual de sobrevivência na área de plantio definitivo

TABLE 1: Percentage of survival in the area of permanent planting.

\begin{tabular}{|c|c|c|c|c|c|c|c|c|c|c|c|}
\hline \multirow{2}{*}{ Bloco } & \multicolumn{2}{|c|}{30 dias } & \multicolumn{5}{|c|}{180 dias } & \multicolumn{4}{|c|}{300 dias } \\
\hline & Mai & Jun & Jul & Ago & Set & Out & Nov & Dez & Jan & $\mathrm{Fev}$ & Mar \\
\hline Adubado Coroado (AC) & \multicolumn{2}{|c|}{$78 \%$} & \multicolumn{5}{|c|}{$78 \%$} & \multicolumn{4}{|c|}{$74 \%$} \\
\hline Adubado Terra Nua (ATN) & \multicolumn{2}{|c|}{$88 \%$} & \multicolumn{5}{|c|}{$88 \%$} & \multicolumn{4}{|c|}{$72 \%$} \\
\hline Sem Adubo Coroado (BC) & \multicolumn{2}{|c|}{$92 \%$} & \multicolumn{5}{|c|}{$92 \%$} & \multicolumn{4}{|c|}{$88 \%$} \\
\hline Sem Adubo Terra Nua (BTN) & \multicolumn{2}{|c|}{$92 \%$} & \multicolumn{5}{|c|}{$92 \%$} & \multicolumn{4}{|c|}{$84 \%$} \\
\hline
\end{tabular}

Nos estágios iniciais de desenvolvimento da cultura, antes de ocorrer o fechamento do dossel, a competição por recursos do solo é, comparativamente, mais importante do que aquela que ocorre por radiação solar, uma vez que ainda não há essa limitação de modo a causar prejuízo ao crescimento das plantas (SEMERE e FROUD-WILLIAMS, 2001). No entanto, em estágios mais avançados de desenvolvimento, a competição por radiação é mais expressiva (SEMERE e FROUD-WILLIAMS, 2001). Nesse trabalho, o crescimento da vegetação nas entrelinhas dos blocos $\mathrm{AC}$ e $\mathrm{BC}$ não foi suficiente para sombrear as mudas, durante o tempo de duração deste trabalho, de tal forma que impedisse $\mathrm{o}$ acesso à radiação solar.

As condições meteorológicas durante os primeiros 30 dias de plantio podem ter influenciado na média da mortalidade inicial das plantas (12,5\%), provavelmente porque saíram de uma condição de sombra e regas diárias e foram plantadas a céu aberto, onde $60,1 \mathrm{~mm}$ de precipitação ocorreram neste mesmo período. Aos 180 dias do plantio a mortalidade foi nula. Neste período a média mensal do índice pluviométrico foi de $130,74 \mathrm{~mm}$.

A média do percentual de sobrevivência dos blocos nos 180 dias subsequentes ao plantio foi de $87,5 \%$ acima do verificado pelo Instituto de Pesquisas e Estudos Florestais (IPEF, 2010) para esta espécie, onde a média nesse mesmo período foi de $75 \%$. Enquanto que a média de sobrevivência aos 300 dias de plantio foi de $79,5 \%$, portanto maior do que a média da mortalidade nos blocos aos 180 dias.

As mudas plantadas em locais definitivos que sofreram diferentes tipos de tratamentos (Figuras 1, 2, 3 e 4) foram submetidas à análise do incremento de seu crescimento, os cálculos estatísticos referentes ao desempenho da espécie estão representados na Tabela 2.

No tratamento $\mathrm{BC}$, o crescimento médio das mudas de Cordia trichotoma foi inferior às demais maneiras de cultivo ao longo dos 300 dias de tratamentos. As taxas de crescimento de um vegetal podem refletir a habilidade e a hierarquia competitiva das espécies na comunidade vegetal (ROUSH e RADOSEVICH, 1985) indicando que o crescimento de $\mathrm{BC}$ provavelmente pode ter sido influenciado por este fator, além da falta de adubação.

Antes do início do tratamento com adubação, verificou-se que os dois blocos AC e BC apresentaram crescimento diferenciado, podendo indicar que o comportamento com ausência de competição gera investimento em crescimento e amplifica as diferenças de potencial genético.

Aos 210 dias, o bloco BTN apresentou crescimento médio inferior aos outros períodos de tratamentos, pois $30 \%$ das arvoretas foram cortadas por formigas o que pode ter interferido no crescimento, alterando a diferença entre a média de crescimento neste período, entre BTN e ATN em relação aos outros períodos. Ao se comparar as médias aos 300 dias percebe-se que ATN e BTN apresentaram desempenho semelhante podendo indicar que o comportamento com ausência de competição gera investimento em crescimento. $\mathrm{O}$ incremento dos blocos de terra nua foram maiores que os coroados e, no caso de AC, pode ter sido superior ao bloco $\mathrm{BC}$, porque recebeu adubação química.

$O$ coeficiente de variabilidade mede a variabilidade dos dados em relação à média e quanto menor o coeficiente mais homogêneo é o conjunto de dados. A análise do coeficiente de variabilidade apresentado na Tabela 2 mostra que a distribuição dos incrementos não segue o padrão da distribuição normal, constatando-se a presença de outliers - valores atípicos ou extremos que estão fora da amplitude dos quartis dos dados, podendo ser observados especialmente em BC com 120 e 210 dias.

Este fato pode ser observado ainda, pela análise do desvio padrão que mede a dispersão dos valores de uma distribuição normal em relação à sua média. Valores altos para o desvio padrão ocorrem quando os dados que estão sendo analisados 
TABELA 2: Estatística descritiva dos incrementos de crescimento em altura $(\mathrm{cm})$ de Cordia trichotoma ao longo de 300 dias sob quatro diferentes maneiras de cultivo.

TABLE 2: Descriptive statistics of the increments of height growth $(\mathrm{cm})$ of Cordia trichotoma over 300 days under four different cultures.

\begin{tabular}{|c|c|c|c|c|c|c|c|c|c|c|c|c|}
\hline \multirow{3}{*}{$\begin{array}{c}\begin{array}{c}\text { Dias depois do } \\
\text { plantio (DDP) }\end{array} \\
\\
\text { BLOCOS }\end{array}$} & \multicolumn{4}{|c|}{120} & \multicolumn{4}{|c|}{210} & \multicolumn{4}{|c|}{300} \\
\hline & \multicolumn{4}{|c|}{ Jul./Set } & \multicolumn{4}{|c|}{ Out./Dez } & \multicolumn{4}{|c|}{ Jan./Mar } \\
\hline & ATN & BTN & $\mathrm{AC}$ & $\mathrm{BC}$ & ATN & BTN & $\mathrm{AC}$ & $\mathrm{BC}$ & ATN & BTN & $\mathrm{AC}$ & $\mathrm{BC}$ \\
\hline Média & 7,36 & 7,82 & 7,9 & 3,64 & 13,26 & 6,88 & 7,06 & 5,62 & 15,12 & 14,18 & 12 & 8,04 \\
\hline Erro Padrão (EP) & 1,22 & 1,40 & 1,21 & 0,80 & 1,89 & 0,87 & 0,96 & 0,61 & 2,08 & 2,14 & 1,72 & 1,30 \\
\hline Mediana & 6 & 3 & 5,5 & 1 & 11,5 & 5 & 6 & 6 & 15 & 10 & 10,5 & 6,5 \\
\hline Moda & 0 & 2 & 0 & 0 & 0 & 0 & 0 & 0 & 0 & 0 & 0 & 0 \\
\hline Desvio Padrão (DP) & 8,60 & 9,91 & 8,55 & 5,64 & 13,36 & 6,16 & 6,82 & 4,34 & 14,70 & 15,16 & 12,18 & 9,19 \\
\hline Variância & 73,99 & 98,27 & 73,11 & 31,83 & 178,56 & 37,94 & 46,55 & 18,81 & 215,98 & 229,94 & 148,36 & 84,53 \\
\hline Curtose & 1,74 & 1,99 & 0,78 & 8,80 & $-0,44$ & $-0,73$ & $-0,30$ & $-0,78$ & $-1,11$ & 1,21 & $-1,10$ & 0,95 \\
\hline Assimetria & 1,54 & 1,66 & 1,16 & 2,58 & 0,73 & 0,61 & 0,68 & 0,27 & 0,45 & 1,27 & 0,50 & 1,20 \\
\hline Intervalo & 32 & 38 & 34 & 30 & 44 & 21 & 25 & 15 & 44 & 59 & 38 & 35 \\
\hline Mínimo & 0 & 0 & 0 & 0 & 0 & 0 & 0 & 0 & 0 & 0 & 0 & 0 \\
\hline Máximo & 32 & 38 & 34 & 30 & 44 & 21 & 25 & 15 & 44 & 59 & 38 & 35 \\
\hline Contagem & 50 & 50 & 50 & 50 & 50 & 50 & 50 & 50 & 50 & 50 & 50 & 50 \\
\hline $\begin{array}{l}\text { Coeficiente de } \\
\text { Variabilidade }\end{array}$ & 116,86 & 126,76 & 108,22 & 2154,97 & 100,76 & 89,52 & 96,62 & 77,17 & 97,19 & 106,93 & 101,50 & 114,34 \\
\hline $\begin{array}{l}\text { Nível de Confiança } \\
\text { (NC) }(95 \%)\end{array}$ & 2,44 & 2,82 & 2,43 & 1,60 & 3,80 & 1,75 & 1,94 & 1,23 & 4,18 & 4,31 & 3,46 & 2,61 \\
\hline
\end{tabular}

estão dispersos em relação à média, como ocorrido em ATN com 210 e 300 dias e também BTN com 300 dias. Do mesmo modo, valores baixos para o desvio padrão ocorrem quando o incremento observado no crescimento da espécie analisada está mais homogêneo em relação à média, como ocorreu em $\mathrm{BC}$ ao longo do período analisado. Esta variabilidade de medidas pode ter origem genética, porque aos 120 dias os blocos ainda não estavam adubados, não sendo este fator, portanto, responsável pela dispersão. A presença desses valores extremos pode significar alta heterogeneidade genética, apontando para a possibilidade de seleção com vistas ao melhoramento genético, possibilitando o estabelecimento de plantios mais homogêneos (WENDLING et al., 2005).

O número de plantas sobreviventes na área de estudo deverá ser suficiente para formar um dossel compacto e satisfatório. De acordo com Roman et al. (2009), o espaço médio ocupado pelos indivíduos de uma população de Cordia trichotoma foi de aproximadamente $15 \mathrm{~m}^{2}$, com variação entre 1,9 e $43 \mathrm{~m}^{2}$, dada a grande amplitude dimensional das árvores. Segundo o IPEF (2010), os melhores incrementos volumétricos registrados em plantios com esta espécie são $9,65 \mathrm{~m}^{3} / \mathrm{ha}$./ano aos cinco anos de idade, usando espaçamento médio de $2 \times 3 \mathrm{~m}$. Embora a formação de um dossel compacto seja possível, a heterogeneidade no crescimento pode tornar inviável seu plantio comercial, pois a formação de árvores com fustes baixos e morfologia inadequada pode ocasionar pouco interesse comercial (MANTOVANI et al., 2001).

Tal como relatado por Carvalho (1988a), as mudas de Cordia trichotoma se apresentaram desuniforme, tanto em altura como em diâmetro, possivelmente necessitando que os plantios sejam realizados em terrenos de média a alta fertilidade, como ocorre também com Cordia superba, do mesmo gênero, e outras espécies florestais nativas (MELO et al., 2004).

\section{CONCLUSÕES}

Cordia trichotoma apresenta alta sobrevivência ao sistema de transplante de canteiro para sacos de mudas com poda da raiz pivotante. O bloco Sem Adubo Coroado (BC) apresentou o maior percentual de sobrevivência, porém, os menores incrementos em altura, podendo exigir menos inves- 
timento financeiro neste método de plantio, sendo uma boa opção para regeneração de locais degradados e mata ciliar, onde a sobrevivência das mudas pode ser mais interessante que a velocidade de crescimento.

Eliminando-se parcialmente a competição através de coroamento das mudas e adubando-as, bloco AC, a média do crescimento encontrado assemelha-se aos blocos de terra nua, ATN e BTN. No entanto, o custo financeiro da operação de coroamento e adição de adubo pode ser menor do que manter toda terra sem cobertura vegetal e livre de competição entre a cultura e outras plantas, além de sugerir ser ambientalmente mais adequado para evitar erosão, ciclagem de nutrientes, proteção, cobertura do solo, entre outros, sugerindo, portanto, estudos posteriores abrangendo estes aspectos.

\section{REFERÊNCIAS BIBLIOGRÁFICAS}

ALMEIDA, M. L.; MUNDSTOCK, C. M. A qualidade da luz afeta o afilhamento em plantas de trigo quando cultivadas sob competição. Ciência Rural, v. 31, n.3, p. 401-408, 2001.

BARBOSA, L. M. Manual sobre princípios de recuperação vegetal de áreas degradadas. São Paulo: Ed. SMA/CEAM/CIMP, 2000. p.76.

BALLARÉ, C. L.; CASAL, J. J. Light signals perceived by crop and weed plants. Field Crops Research, v. 67, n. 2, p. 149-160, 2000.

CARVALHO, P. E. R. Louro-pardo. Colombo: Embrapa Florestas, 1988a. p. 63-66. (Boletim de Pesquisa Florestal, n. 17).

CARVALHO, P. E. R. Potencialidade e restrições da regeneração artificial de espécies madeireiras nativas no Paraná. In: CONGRESSO FLORESTAL DO PARANÁ, 1988, Curitiba. Anais dos resumos... Curitiba, Instituto Florestal do Paraná, 1988b. p. 23. CRESTANA, M. S. M. Árvores \& Cia. Campinas: CATI, 2007. p. 132.

EMPRESA BRASILEIRA DE PESQUISA AGROPECUÁRIA. Centro Nacional de Pesquisa de Florestas, Curitiba, PR Zoneamento ecológico para plantios florestais no Estado do Paraná. Brasília, EMBRAPA-DDT, 1986. p. 89. (Documentos, 17).

GOMES, F. P. Estatística aplicada a experimentos agronômicos e florestais. 1.ed., São Paulo: Ed. Fealq, 2002. 309 p.

IPEF - INSTITUTO DE PESQUISA E ESTUDOS FLORESTAIS, BRASIL Identificação de Espécies

Florestais. Disponível em: <(http://www.ipef.br/ identificacao/nativas/detalhes.asp? $\operatorname{codigo}=25)>$

Acesso em: 20 de outubro de 2010.

LORENZI, H. Árvores Brasileiras: Manual de identificação e cultivo de plantas arbóreas nativas do Brasil. 4. ed. São Paulo: Ed. Nova Odessa, Instituto Plantarum, 2002. v. 1, p. 367.

MANTOVANI, N. C.; FRANCO, E. T. H.; VESTENA, S. Regeneração in vitro de louro-pardo (Cordia trichotoma (Vellozo) Arrabida ex Steudel).

Ciência Florestal, v. 1, n. 2, p. 93-101, 2001.

MATO GROSSODOSUL.Zoneamento Ecológico-

Econômico de Mato Grosso do Sul. Contribuições técnicas, teóricas jurídicas e metodológicas, Campo Grande, 2009. v. 1.

MELO, A. C. G.; DURIGAN, G.; KAWABATA, M. Crescimento e sobrevivência de espécies arbóreas plantadas em áreas de cerrado, AssisSP. In: VILAS BÔAS, O.; DURIGAN, G. (Org.). Resultados da cooperação Brasil/Japão. São Paulo: Páginas \& Letras, 2004. p. 315-324.

MELO, M. D.; SILVA, R. L. C.; QUEIROZ, M. T. M. Levantamento das plantas medicinais na praia de Pitangui, Extremoz. In: CONGRESSO NACIONAL DE BOTÂNICA, 49., 1998, Salvador. Resumos. Salvador: Universidade Federal da Bahia / Instituto de Biologia, 1998, p. 284.

RADOSEVICH, S. R; HOLT, J.; GHERSA, C. Weed ecology: implications for management. 2nd. ed. New York: Willey, 1997. 589 p.

ROMAN, M.; BRESSAN, D. A.; DURLO, M. A. Variáveis morfométricas e relações interdimensionais para Cordia trichotoma (Vell.) Arráb. ex Steud. Ciência Florestal, v. 19, n. 4, p. 473-480, 2009.

ROUSH, M. L.; RADOSEVICH, S. R. Relationships between growth and competitiveness of four annual weeds. Journal of Applied Ecology, v. 22, p. 895905, 1985

Secretaria de Meio Ambiente, do Planejamento, da Ciência e Tecnologia - SEMAC - Plano Estadual de Recursos Hídricos de Mato Grosso do Sul. 2010. Disponível em <http://www.semac.ms.gov. br/>. Acesso em: 18 de março de 2010.

SEMERE, T.; FROUD-WILLIAMS, R. J. The effect of pea cultivar and water stress on root and shoot competition between vegetative plants of maize and pea. Journal of Applied Ecology, v. 38, p. 137-145, 2001.

SMITH, L. B. Boragináceas. In: REITZ, R. Flora ilustrada Catarinense. Itajaí: Herbário BARBOSA Rodrigues, 1970. p.19-20.

SOUZA, P. A.; VENTURIN, N; MACEDO R. 
L. G. Adubação mineral do Ipê-roxo (Tabebuia 223 p. impetinosa), Ciência Florestal, v. 16, n. 3, p. 261- SCHEEREN, L. W.; SCHNEIDER, P. S. P.; 270, 2006. FINGER, C. A. G. Crescimento do Louro-Pardo, WENDlinG, I.; PAIVA, H. N.; GONÇALVES, Cordia trichotoma (Vell.) Arrab. Ex Steud., na W. Técnicas de produção de mudas de plantas ornamentais. Viçosa: Aprenda Fácil, 2005, v. 3, depressão central do Estado do Rio Grande do Sul. Ciência Florestal, v. 12, n. 2, p. 169-176, 2002. 